\title{
Hazardous Gas Detection using Gas Sensors Arrays and Fuzzy-Based Classification
}

\author{
Rajina R. Mohamed, M A Mohamed, Wahidah Hashim, Abd. Rahim , M. Syamil Fuad
}

\begin{abstract}
This paper presents hazardous gas detection using gas sensors arrays and fuzzy-based classification. This research is an automation of hazardous gas detection using electronic nose. Gases surround us could either hazard or benefit our health. Gas detection is an important issue, as humans should not breathe in hazardous gases in order to maintain their health. Hence, there must be an indicator to show the hazardous level of certain gases so that people can avoid and minimize the impact on their health. In this paper, hazardous gas detection is implemented by using gas sensor arrays and fuzzy-based classification. A classification for the electronic nose (e-nose) is developed in order to classify gases and determine the level of hazard of gases. The results found that e-nose system is able to differentiate hazardous level of chosen gases which are LP gas and CO gas.
\end{abstract}

Keywords: Hazardous gas, gas sensors, fuzzy classification.

\section{INTRODUCTION}

The main purpose of gas detection systems is to detect any gases in surrounding and measure accurate readings and then provide appropriate action to be taken by the people within that area. In Malaysia, there is no suitable system to detect gases that are considered hazardous to human health and flammable gases, which may lead to unexpected events such as fire or explosions if they are mixed with air in adequate amount. There are many gases that are hazardous, i.e. ammonia, chlorine, sulphur, carbon monoxide, to name a few. However in this research, the selected gases that need to be detected in the environment are carbon monoxide $(\mathrm{CO})$ and liquefied petroleum gas (LPG). Hazardous gas detection will be implemented by using gas sensor arrays and fuzzy-based classification provides precise readings for the two gases as stated earlier. Based on the discussion above, electronic nose has been used in many fields especially in detecting gas at certain area, condition or environment. Many research has shown detection of gases using e-nose in many fields, and using various methods of classifications. By implementing fuzzy logic classification on the electronic nose system, the hazardous level of gases can be determined.

\section{II.SYSTEM DESIGN}

Electronic nose is an embedded-based system to help distinguish the hazardous level of gases by using the following mechanism; the e-nose sensor arrays will detect the gases surrounding the area, then the sensor arrays will respond in electrical signal form. Then, the value will be transmitted to the signal processing unit to perform the analysis of the sampling pattern in order to determine the hazardous level of the gases detected.

\section{A. E-Nose System Description}

The combination of e-nose hardware and the classification will generate precise output. The system will determine whether the gases are dangerous once the sensor arrays respond to them. After the digital signal from the sensor is sent to the microcontroller, it will be computed and the dangerous level of gases will be determined by using the developed classification method. The dangerous level will be displayed on the LCD monitor. There are few electronics parts that will be integrated in this system such as breadboard, LCD monitor and sensors in order to be functioned as gas detector.

\section{B. Flowchart}

Figure 1 shows the flow chart of the system. Based on the Figure 1, the e-nose system will be having a condition whereby if the detectable gas is not present, it will go to initial step. If the gas is present, it will respond and sends the digital signal to the microcontroller. Microcontroller then will compute the signal, by using the developed gas classification method, and then will determine the hazardous level of gas detected.

\footnotetext{
Revised Version Manuscript Received on August 19, 2019.

Rajina R. Mohamed, Department of Computing, College of Computing and Informatic, niversity Tenaga Nasional, Malaysia. (email:rajina@uniten.edu.my)

M A Mohamed, Universiti Sultan Zainal Abidin.(email: mafendee@unisza@edu.my)

Wahidah Hashim , Institute of Informatics and Computing Energy, University Tenaga Nasional, Malaysia(email:wahidah@uniten.edu.my)

Abd Rahim Ahmad, Department of Computing, College of Computing and Informatic, niversity Tenaga Nasional, Malaysia(email: abdrahim@uniten.edu.my)

M. Syamil Fuad, Institute of Informatics and Computing Energy, University Tenaga Nasional, Malaysia(email: syamilfuad@uniten.edu.my)
} 


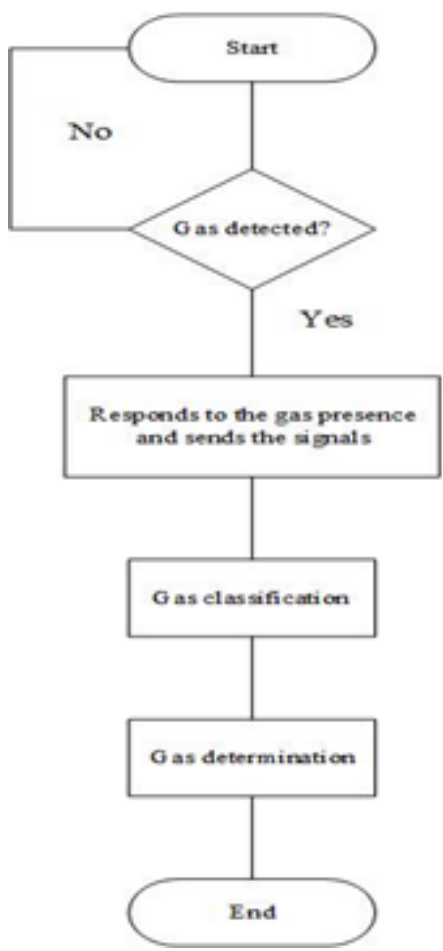

Figure 1: System flow chart

\section{C.System Design}

The system was designed to contain a various components that fulfill the research purpose. Figure 2 shows the system design of e-nose system.

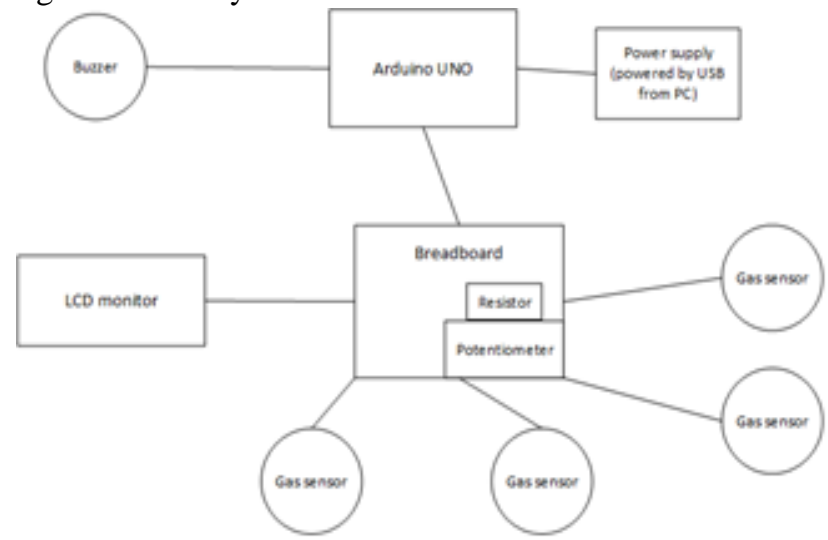

Figure 2: System design of electronic nose

Based on Figure 2, Arduino board will be powered by direct connection through USB cable or optionally, with batteries. Once it is powered on, the gas sensors needs to be placed in the area where the gases (CO and LPG) are present. For testing purpose, this process will be done inside a confined space. The gas sensors will react to the presence of the gases and the microcontroller will do its task as required. The output will be displayed on the LCD monitor.

\section{III.IMPLEMENTATION}

In this section, it will describe systematically on how to set up the e-nose system for the hardware as well as the testing and results from the fuzzy classifier.

\section{A. Hardware Component Development}

All the listed hardware components will be assembled and connected to the microcontroller board, which is the Arduino Uno. To develop the prototype device, the listed items which include Arduino Uno, Breadboard, Buzzer, Jumper wires, LCD monitor (20x4), MQ-6 gas sensor (LPG, propane gas), MQ-7 gas sensor (Carbon monoxide gas), Potentiometer, Resistor.

\section{B. Classification}

Figure 3 shows the flow chart of fuzzy logic designing.

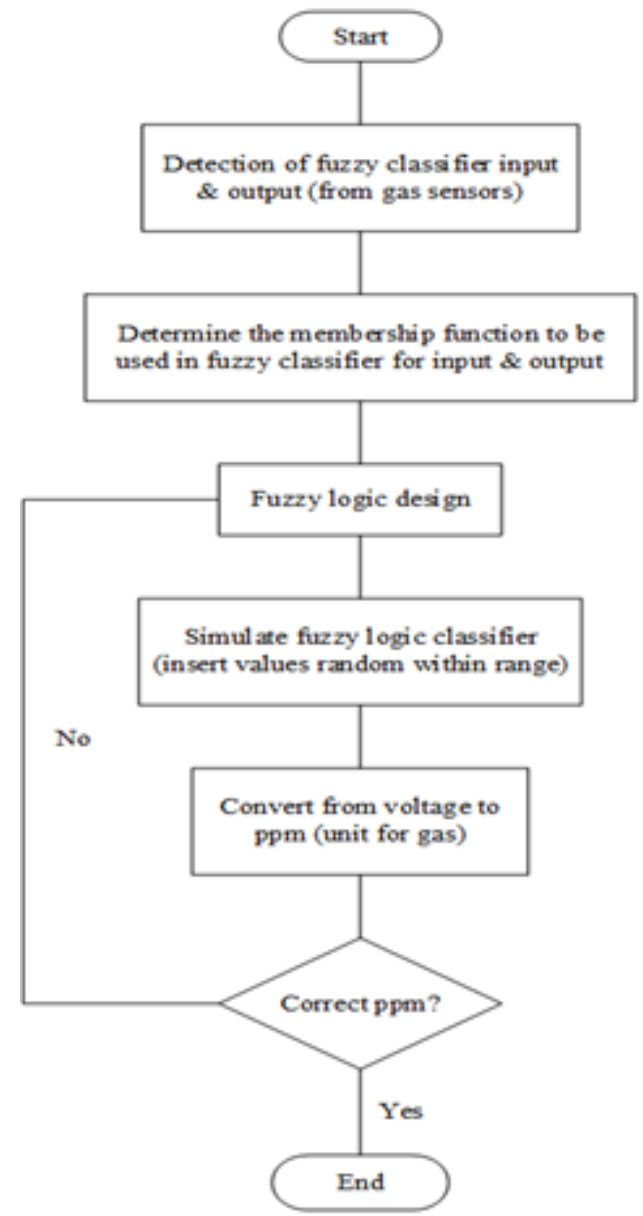

Figure 3: Fuzzy Logic Designing Flow Chart

\section{Fuzzification}

Fuzzification is the process of changing a real scalar value into a fuzzy value. This process is achieved by using the different types of membership functions (fuzzifiers). The first step is to take the inputs from MQ-6 and MQ-7 sensors and determine the degree to which inputs belong to each of the fuzzy set.

The inputs are listed as below:

1st sensor: MQ-6 $\square$ Voltage = fuzzy set $[\mathrm{LOW}$, MEDIUM, HIGH], range [0-5]

2nd sensor: MQ-7 $\square$ Voltage = fuzzy set [LOW, MEDIUM, HIGH], range [0-5]

3rd sensor: MQ-6 $\square$ Voltage = fuzzy set [LOW, MEDIUM, HIGH], range [0-5]

4th sensor: MQ-7 $\square$ Voltage = fuzzy set $[\mathrm{LOW}$, MEDIUM, HIGH], range [0-5]

\section{Published By:}

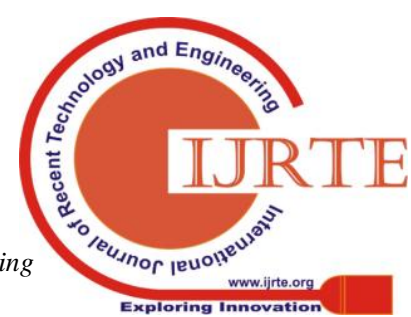


International Journal of Recent Technology and Engineering (IJRTE) ISSN: 2277-3878, Volume-8 Issue-2S8, August 2019

The output is listed as below:

Result: Level $=\{$ SAFE, LESS SAFE, DANGERUS $\}$, range $\{0-800\}$

\section{Fuzzy Rules}

The next step is to use the inputs from all three sensors and apply them to the antecedents of the fuzzy rules. The 'AND' operation is used to obtain a single value that represents the result of the antecedent evaluation. This number is then applied to the consequence membership function. Table $1-3$ shows all 81 rules belong to the system.

Table 1: Fuzzy Rules (Rule 1 - 27)

\begin{tabular}{|c|c|c|c|c|c|}
\hline $\begin{array}{l}\text { Number } \\
\text { of Rules }\end{array}$ & $\begin{array}{c}1^{\text {st }} \\
\text { Sensor } \\
\text { (MQ-6) }\end{array}$ & $\begin{array}{c}2^{\text {nd }} \text { Sensor } \\
\text { (MQ-7) }\end{array}$ & $\begin{array}{c}3^{\text {rd }} \text { Sensor } \\
\text { (MQ-6) }\end{array}$ & $\begin{array}{c}4^{\text {th }} \text { Sensor } \\
\text { (MQ-7) }\end{array}$ & Result \\
\hline 1 & LOW & LOW & LOW & LOW & SAFE \\
\hline 2 & LOW & LOW & LOW & MEDIUM & SAFE \\
\hline 3 & LOW & LOW & LOW & HIGH & SAFE \\
\hline 4 & LOW & LOW & MEDIUM & LOW & SAFE \\
\hline 5 & LOW & LOW & MEDIUM & MEDIUM & SAFE \\
\hline 6 & LOW & LOW & MEDIUM & HIGH & SAFE \\
\hline 7 & LOW & LOW & HIGH & LOW & SAFE \\
\hline 8 & LOW & LOW & HIGH & MEDIUM & LESS SAFE \\
\hline 9 & LOW & LOW & HIGH & HIGH & DANGEROUS \\
\hline 10 & LOW & MEDIUM & LOW & LOW & SAFE \\
\hline 11 & LOW & MEDIUM & LOW & MEDIUM & SAFE \\
\hline 12 & LOW & MEDIUM & LOW & HIGH & LESS SAFE \\
\hline 13 & LOW & MEDIUM & MEDIUM & LOW & SAFE \\
\hline 14 & LOW & MEDIUM & MEDIUM & MEDIUM & LESS SAFE \\
\hline 15 & LOW & MEDIUM & MEDIUM & HIGH & LESS SAFE \\
\hline 16 & LOW & MEDIUM & HIGH & LOW & SAFE \\
\hline 17 & LOW & MEDIUM & HIGH & MEDIUM & LESS SAFE \\
\hline 18 & LOW & MEDIUM & HIGH & HIGH & DANGEROUS \\
\hline 19 & LOW & HIGH & LOW & LOW & SAFE \\
\hline 20 & LOW & HIGH & LOW & MEDIUM & SAFE \\
\hline 21 & LOW & $\mathrm{HIGH}$ & LOW & HIGH & DANGEROUS \\
\hline 22 & LOW & $\mathrm{HIGH}$ & MEDIUM & LOW & LESS SAFE \\
\hline 23 & LOW & HIGH & MEDIUM & MEDIUM & LESS SAFE \\
\hline 24 & LOW & HIGH & MEDIUM & HIGH & DANGEROUS \\
\hline 25 & LOW & HIGH & HIGH & LOW & DANGEROUS \\
\hline 26 & LOW & HIGH & HIGH & MEDIUM & DANGEROUS \\
\hline 27 & LOW & HIGH & HIGH & HIGH & DANGEROUS \\
\hline
\end{tabular}

Table 2: Fuzzy Rules (Rule 28 - 54)

\begin{tabular}{|c|c|c|c|c|c|}
\hline $\begin{array}{l}\text { Num } \\
\text { ber of } \\
\text { Rules }\end{array}$ & $\begin{array}{l}1^{\text {st }} \\
\text { Sensor } \\
\text { (MQ-6 } \\
\text { ) }\end{array}$ & $\begin{array}{l}2^{\text {nd }} \\
\text { Sensor } \\
\text { (MQ-7 } \\
\text { ) }\end{array}$ & $\begin{array}{l}3^{\text {rd }} \\
\text { Sensor } \\
\text { (MQ-6 } \\
\text { ) }\end{array}$ & $\begin{array}{l}4^{\text {th }} \\
\text { Sensor } \\
\text { (MQ-7 } \\
\text { (M) }\end{array}$ & Result \\
\hline 28 & $\begin{array}{l}\text { MEDI } \\
\text { UM }\end{array}$ & LOW & LOW & LOW & SAFE \\
\hline 29 & $\begin{array}{l}\text { MEDI } \\
\text { UM }\end{array}$ & LOW & LOW & $\begin{array}{l}\text { MEDI } \\
\text { UM }\end{array}$ & SAFE \\
\hline 30 & $\begin{array}{l}\text { MEDI } \\
\text { UM }\end{array}$ & LOW & LOW & HIGH & SAFE \\
\hline 31 & $\begin{array}{l}\text { MEDI } \\
\text { UM } \\
\end{array}$ & LOW & $\begin{array}{l}\text { MEDI } \\
\text { UM }\end{array}$ & LOW & SAFE \\
\hline 32 & $\begin{array}{l}\text { MEDI } \\
\text { UM }\end{array}$ & LOW & $\begin{array}{l}\text { MEDI } \\
\text { UM }\end{array}$ & $\begin{array}{l}\text { MEDI } \\
\text { UM }\end{array}$ & $\begin{array}{l}\text { LESS } \\
\text { SAFE }\end{array}$ \\
\hline 33 & $\begin{array}{l}\text { MEDI } \\
\text { UM }\end{array}$ & LOW & $\begin{array}{l}\text { MEDI } \\
\text { UM }\end{array}$ & HIGH & $\begin{array}{l}\text { LESS } \\
\text { SAFE }\end{array}$ \\
\hline 34 & $\begin{array}{l}\text { MEDI } \\
\text { UM }\end{array}$ & LOW & HIGH & LOW & SAFE \\
\hline 35 & $\begin{array}{l}\text { MEDI } \\
\text { UM }\end{array}$ & LOW & HIGH & $\begin{array}{l}\text { MEDI } \\
\text { UM } \\
\end{array}$ & $\begin{array}{l}\text { LESS } \\
\text { SAFE } \\
\end{array}$ \\
\hline
\end{tabular}

\begin{tabular}{|c|c|c|c|c|c|}
\hline 36 & $\begin{array}{l}\text { MEDI } \\
\text { UM }\end{array}$ & LOW & HIGH & HIGH & $\begin{array}{l}\text { DANGER } \\
\text { OUS }\end{array}$ \\
\hline 37 & $\begin{array}{l}\text { MEDI } \\
\text { UM }\end{array}$ & $\begin{array}{l}\text { MEDI } \\
\text { UM }\end{array}$ & LOW & LOW & SAFE \\
\hline 38 & $\begin{array}{l}\text { MEDI } \\
\text { UM }\end{array}$ & $\begin{array}{l}\text { MEDI } \\
\text { UM }\end{array}$ & LOW & $\begin{array}{l}\text { MEDI } \\
\text { UM }\end{array}$ & SAFE \\
\hline 39 & $\begin{array}{l}\text { MEDI } \\
\text { UM }\end{array}$ & $\begin{array}{l}\text { MEDI } \\
\text { UM }\end{array}$ & LOW & HIGH & $\begin{array}{l}\text { LESS } \\
\text { SAFE }\end{array}$ \\
\hline 40 & $\begin{array}{l}\text { MEDI } \\
\text { UM }\end{array}$ & $\begin{array}{l}\text { MEDI } \\
\text { UM }\end{array}$ & $\begin{array}{l}\text { MEDI } \\
\text { UM }\end{array}$ & LOW & $\begin{array}{l}\text { LESS } \\
\text { SAFE }\end{array}$ \\
\hline 41 & $\begin{array}{l}\text { MEDI } \\
\text { UM }\end{array}$ & $\begin{array}{l}\text { MEDI } \\
\text { UM }\end{array}$ & $\begin{array}{l}\text { MEDI } \\
\text { UM }\end{array}$ & $\begin{array}{l}\text { MEDI } \\
\text { UM }\end{array}$ & $\begin{array}{l}\text { LESS } \\
\text { SAFE }\end{array}$ \\
\hline 42 & $\begin{array}{l}\text { MEDI } \\
\text { UM }\end{array}$ & $\begin{array}{l}\text { MEDI } \\
\text { UM }\end{array}$ & $\begin{array}{l}\text { MEDI } \\
\text { UM }\end{array}$ & HIGH & $\begin{array}{l}\text { LESS } \\
\text { SAFE }\end{array}$ \\
\hline 43 & $\begin{array}{l}\text { MEDI } \\
\text { UM }\end{array}$ & $\begin{array}{l}\text { MEDI } \\
\text { UM }\end{array}$ & HIGH & LOW & $\begin{array}{l}\text { LESS } \\
\text { SAFE }\end{array}$ \\
\hline 44 & $\begin{array}{l}\text { MEDI } \\
\text { UM }\end{array}$ & $\begin{array}{l}\text { MEDI } \\
\text { UM }\end{array}$ & HIGH & $\begin{array}{l}\text { MEDI } \\
\text { UM }\end{array}$ & $\begin{array}{l}\text { LESS } \\
\text { SAFE }\end{array}$ \\
\hline 45 & $\begin{array}{l}\text { MEDI } \\
\text { UM }\end{array}$ & $\begin{array}{l}\text { MEDI } \\
\text { UM }\end{array}$ & HIGH & HIGH & $\begin{array}{l}\text { DANGER } \\
\text { OUS }\end{array}$ \\
\hline 46 & $\begin{array}{l}\text { MEDI } \\
\text { UM }\end{array}$ & HIGH & LOW & LOW & $\begin{array}{l}\text { LESS } \\
\text { SAFE }\end{array}$ \\
\hline 47 & $\begin{array}{l}\text { MEDI } \\
\text { UM }\end{array}$ & HIGH & LOW & $\begin{array}{l}\text { MEDI } \\
\text { UM }\end{array}$ & $\begin{array}{l}\text { DANGER } \\
\text { OUS }\end{array}$ \\
\hline 48 & $\begin{array}{l}\text { MEDI } \\
\text { UM }\end{array}$ & HIGH & LOW & HIGH & $\begin{array}{l}\text { DANGER } \\
\text { OUS }\end{array}$ \\
\hline 49 & $\begin{array}{l}\text { MEDI } \\
\text { UM }\end{array}$ & HIGH & $\begin{array}{l}\text { MEDI } \\
\text { UM }\end{array}$ & LOW & $\begin{array}{l}\text { LESS } \\
\text { SAFE }\end{array}$ \\
\hline 50 & $\begin{array}{l}\text { MEDI } \\
\text { UM }\end{array}$ & HIGH & $\begin{array}{l}\text { MEDI } \\
\text { UM }\end{array}$ & $\begin{array}{l}\text { MEDI } \\
\text { UM }\end{array}$ & $\begin{array}{l}\text { DANGER } \\
\text { OUS }\end{array}$ \\
\hline 51 & $\begin{array}{l}\text { MEDI } \\
\text { UM }\end{array}$ & HIGH & $\begin{array}{l}\text { MEDI } \\
\text { UM }\end{array}$ & HIGH & $\begin{array}{l}\text { DANGER } \\
\text { OUS }\end{array}$ \\
\hline 52 & $\begin{array}{l}\text { MEDI } \\
\text { UM }\end{array}$ & HIGH & HIGH & LOW & $\begin{array}{l}\text { DANGER } \\
\text { OUS }\end{array}$ \\
\hline 53 & $\begin{array}{l}\text { MEDI } \\
\text { UM }\end{array}$ & HIGH & HIGH & $\begin{array}{l}\text { MEDI } \\
\text { UM }\end{array}$ & $\begin{array}{l}\text { DANGER } \\
\text { OUS }\end{array}$ \\
\hline 54 & $\begin{array}{l}\text { MEDI } \\
\text { UM }\end{array}$ & HIGH & HIGH & HIGH & $\begin{array}{l}\text { DANGER } \\
\text { OUS }\end{array}$ \\
\hline
\end{tabular}


Table 3: Fuzzy Rules (Rule 55 - 81)

\begin{tabular}{|c|c|c|c|c|c|}
\hline $\begin{array}{l}\text { Num } \\
\text { ber of } \\
\text { Rules }\end{array}$ & $\begin{array}{l}1^{\text {st }} \\
\text { Sens } \\
\text { or } \\
(\mathrm{MQ}- \\
6) \\
\end{array}$ & $\begin{array}{l}2^{\text {nd }} \\
\text { Sensor } \\
\text { (MQ-7 } \\
\text { ) }\end{array}$ & $\begin{array}{l}3^{\text {rd }} \\
\text { Sensor } \\
\text { (MQ-6 } \\
\text { ) }\end{array}$ & $\begin{array}{l}4^{\text {th }} \\
\text { Sensor } \\
\text { (MQ-7 } \\
\text { ) }\end{array}$ & Result \\
\hline 55 & $\begin{array}{l}\mathrm{HIG} \\
\mathrm{H}\end{array}$ & LOW & LOW & LOW & SAFE \\
\hline 56 & $\begin{array}{l}\text { HIG } \\
\mathrm{H}\end{array}$ & LOW & LOW & $\begin{array}{l}\text { MEDI } \\
\text { UM }\end{array}$ & SAFE \\
\hline 57 & $\begin{array}{l}\text { HIG } \\
\mathrm{H} \\
\end{array}$ & LOW & LOW & HIGH & $\begin{array}{l}\text { DANGER } \\
\text { OUS }\end{array}$ \\
\hline 58 & $\begin{array}{l}\text { HIG } \\
\mathrm{H}\end{array}$ & LOW & $\begin{array}{l}\text { MEDI } \\
\text { UM }\end{array}$ & LOW & $\begin{array}{l}\text { LESS } \\
\text { SAFE }\end{array}$ \\
\hline 59 & $\begin{array}{l}\text { HIG } \\
\mathrm{H}\end{array}$ & LOW & $\begin{array}{l}\text { MEDI } \\
\text { UM }\end{array}$ & $\begin{array}{l}\text { MEDI } \\
\text { UM }\end{array}$ & $\begin{array}{l}\text { LESS } \\
\text { SAFE }\end{array}$ \\
\hline 60 & $\begin{array}{l}\mathrm{HIG} \\
\mathrm{H} \\
\end{array}$ & LOW & $\begin{array}{l}\text { MEDI } \\
\text { UM }\end{array}$ & HIGH & $\begin{array}{l}\text { DANGER } \\
\text { OUS }\end{array}$ \\
\hline 61 & $\begin{array}{l}\text { HIG } \\
\mathrm{H}\end{array}$ & LOW & HIGH & LOW & $\begin{array}{l}\text { DANGER } \\
\text { OUS }\end{array}$ \\
\hline 62 & $\begin{array}{l}\text { HIG } \\
\mathrm{H}\end{array}$ & LOW & HIGH & $\begin{array}{l}\text { MEDI } \\
\text { UM }\end{array}$ & $\begin{array}{l}\text { DANGER } \\
\text { OUS }\end{array}$ \\
\hline 63 & $\begin{array}{l}\mathrm{HIG} \\
\mathrm{H}\end{array}$ & LOW & HIGH & HIGH & $\begin{array}{l}\text { DANGER } \\
\text { OUS }\end{array}$ \\
\hline 64 & $\begin{array}{l}\mathrm{HIG} \\
\mathrm{H} \\
\end{array}$ & $\begin{array}{l}\text { MEDI } \\
\text { UM }\end{array}$ & LOW & LOW & SAFE \\
\hline 65 & $\begin{array}{l}\text { HIG } \\
\mathrm{H}\end{array}$ & $\begin{array}{l}\text { MEDI } \\
\text { UM }\end{array}$ & LOW & $\begin{array}{l}\text { MEDI } \\
\text { UM }\end{array}$ & $\begin{array}{l}\text { LESS } \\
\text { SAFE }\end{array}$ \\
\hline 66 & $\begin{array}{l}\text { HIG } \\
\mathrm{H}\end{array}$ & $\begin{array}{l}\text { MEDI } \\
\text { UM }\end{array}$ & LOW & HIGH & $\begin{array}{l}\text { DANGER } \\
\text { OUS }\end{array}$ \\
\hline 67 & $\begin{array}{l}\text { HIG } \\
\mathrm{H}\end{array}$ & $\begin{array}{l}\text { MEDI } \\
\text { UM }\end{array}$ & $\begin{array}{l}\text { MEDI } \\
\text { UM }\end{array}$ & LOW & $\begin{array}{l}\text { LESS } \\
\text { SAFE }\end{array}$ \\
\hline 68 & $\begin{array}{l}\mathrm{HIG} \\
\mathrm{H}\end{array}$ & $\begin{array}{l}\text { MEDI } \\
\text { UM }\end{array}$ & $\begin{array}{l}\text { MEDI } \\
\text { UM }\end{array}$ & $\begin{array}{l}\text { MEDI } \\
\text { UM }\end{array}$ & $\begin{array}{l}\text { LESS } \\
\text { SAFE }\end{array}$ \\
\hline 69 & $\begin{array}{l}\text { HIG } \\
\mathrm{H}\end{array}$ & $\begin{array}{l}\text { MEDI } \\
\text { UM }\end{array}$ & $\begin{array}{l}\text { MEDI } \\
\text { UM }\end{array}$ & HIGH & $\begin{array}{l}\text { DANGER } \\
\text { OUS }\end{array}$ \\
\hline 70 & $\begin{array}{l}\text { HIG } \\
\mathrm{H}\end{array}$ & $\begin{array}{l}\text { MEDI } \\
\text { UM }\end{array}$ & HIGH & LOW & $\begin{array}{l}\text { DANGER } \\
\text { OUS }\end{array}$ \\
\hline 71 & $\begin{array}{l}\mathrm{HIG} \\
\mathrm{H}\end{array}$ & $\begin{array}{l}\text { MEDI } \\
\text { UM }\end{array}$ & HIGH & $\begin{array}{l}\text { MEDI } \\
\text { UM }\end{array}$ & $\begin{array}{l}\text { DANGER } \\
\text { OUS }\end{array}$ \\
\hline 72 & $\begin{array}{l}\text { HIG } \\
\mathrm{H} \\
\end{array}$ & $\begin{array}{l}\text { MEDI } \\
\text { UM }\end{array}$ & HIGH & HIGH & $\begin{array}{l}\text { DANGER } \\
\text { OUS }\end{array}$ \\
\hline 73 & $\begin{array}{l}\text { HIG } \\
\mathrm{H}\end{array}$ & HIGH & LOW & LOW & $\begin{array}{l}\text { DANGER } \\
\text { OUS }\end{array}$ \\
\hline 74 & $\begin{array}{l}\mathrm{HIG} \\
\mathrm{H} \\
\end{array}$ & HIGH & LOW & $\begin{array}{l}\text { MEDI } \\
\text { UM }\end{array}$ & $\begin{array}{l}\text { DANGER } \\
\text { OUS }\end{array}$ \\
\hline 75 & $\begin{array}{l}\mathrm{HIG} \\
\mathrm{H}\end{array}$ & HIGH & LOW & HIGH & $\begin{array}{l}\text { DANGER } \\
\text { OUS }\end{array}$ \\
\hline 76 & $\begin{array}{l}\text { HIG } \\
\mathrm{H}\end{array}$ & HIGH & $\begin{array}{l}\text { MEDI } \\
\text { UM }\end{array}$ & LOW & $\begin{array}{l}\text { DANGER } \\
\text { OUS }\end{array}$ \\
\hline 77 & $\begin{array}{l}\mathrm{HIG} \\
\mathrm{H}\end{array}$ & HIGH & $\begin{array}{l}\text { MEDI } \\
\text { UM }\end{array}$ & $\begin{array}{l}\text { MEDI } \\
\text { UM }\end{array}$ & $\begin{array}{l}\text { DANGER } \\
\text { OUS }\end{array}$ \\
\hline 78 & $\begin{array}{l}\text { HIG } \\
\mathrm{H}\end{array}$ & HIGH & $\begin{array}{l}\text { MEDI } \\
\text { UM }\end{array}$ & HIGH & $\begin{array}{l}\text { DANGER } \\
\text { OUS }\end{array}$ \\
\hline 79 & $\begin{array}{l}\text { HIG } \\
\mathrm{H}\end{array}$ & HIGH & HIGH & LOW & $\begin{array}{l}\text { DANGER } \\
\text { OUS }\end{array}$ \\
\hline 80 & $\begin{array}{l}\text { HIG } \\
\mathrm{H}\end{array}$ & $\mathrm{HIGH}$ & HIGH & $\begin{array}{l}\text { MEDI } \\
\text { UM }\end{array}$ & $\begin{array}{l}\text { DANGER } \\
\text { OUS }\end{array}$ \\
\hline 81 & $\begin{array}{l}\text { HIG } \\
\mathrm{H}\end{array}$ & HIGH & HIGH & HIGH & $\begin{array}{l}\text { DANGER } \\
\text { OUS }\end{array}$ \\
\hline
\end{tabular}

Once the codes have been uploaded to the Arduino, the e-nose system is tested by using LP gas and CO gas resource, which is from mini gas stove or lighter.

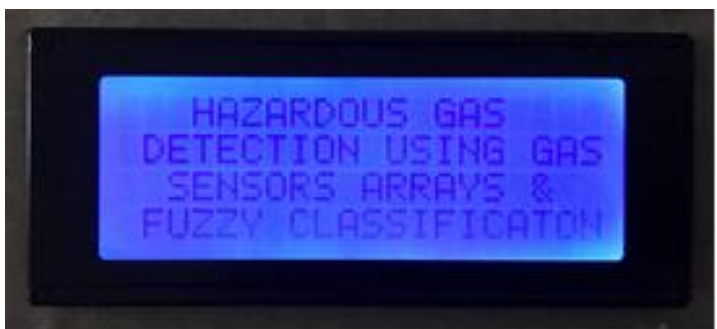

Figure 4: Welcome Screen

Figure 4 shows the welcome screen when the e-nose system is switched on.

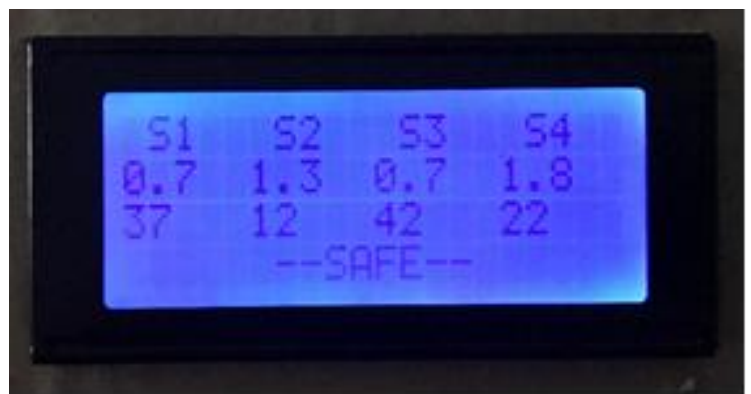

Figure 5: 'Safe' Level Screen

Figure 5 shows the result which is "safe" when the gas sensors are tested with LP gas and/or CO gas with less than $30 \mathrm{ppm}$ and less than $5 \mathrm{ppm}$ respectively.

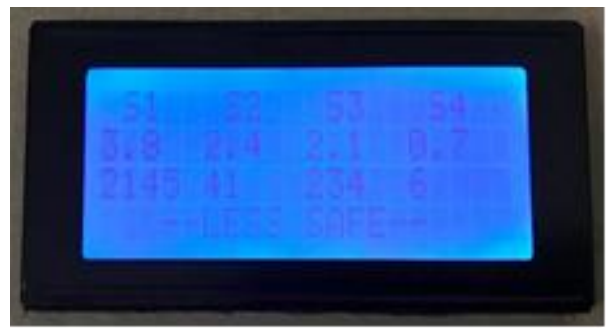

Figure 6: 'Less safe' Level Screen

Figure 6 shows the result which is "less safe" when the gas sensors are tested with LP gas and/or CO gas with more than $30 \mathrm{ppm}$ and more than $5 \mathrm{ppm}$ respectively.

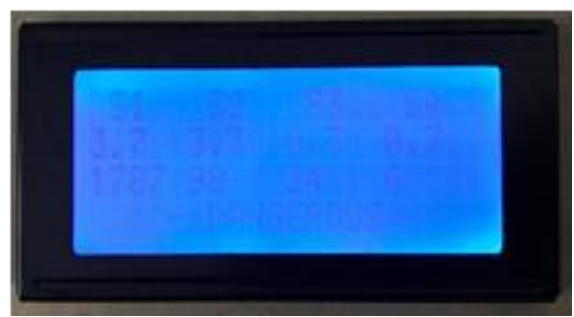

Figure 7: 'Dangerous' Level Screen 
Figure 7 shows the result which is "dangerous" when the gas sensors are tested with LP gas and/or CO gas when they reach more than $800 \mathrm{ppm}$ for LP gas and more than 50ppm for CO gas.

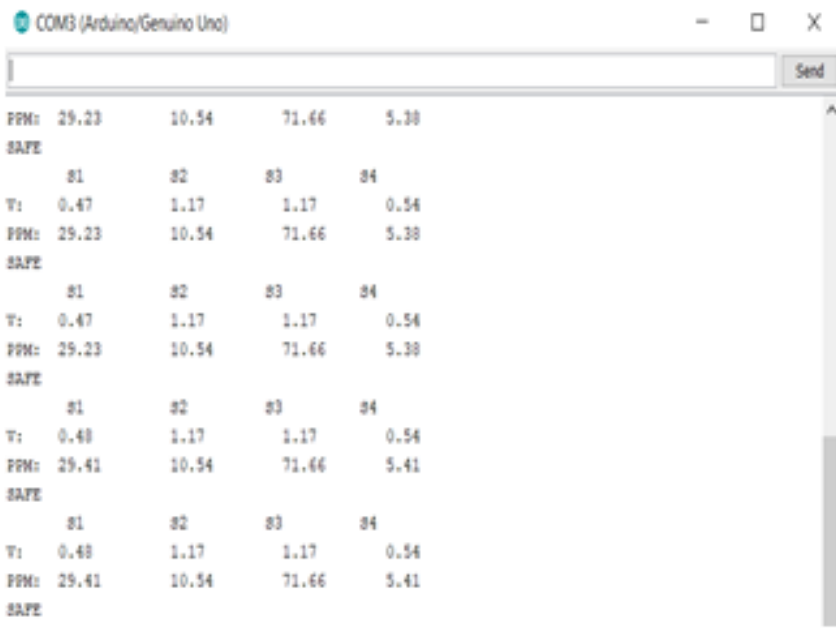

Figure 8: Serial Monitor Readings

Figure 8 shows the similar screen shown by the LCD monitor at serial monitor of Arduino IDE. The purpose of duplicating monitoring screen is to have a backup monitoring screen if the LCD monitor is not working.

In order to verify the classification and output of the e-nose system, the verification is done by comparing the gas of both LP and CO in ppm unit to values that have been set earlier in the research, which summarized in Table 4 and Table 5.

Table 4: Result for LP Gas

\begin{tabular}{|c|l|}
\hline Value (ppm) & Classification result \\
\hline Less than 30ppm & Safe \\
\hline $\begin{array}{l}\text { In between 30ppm \& } \\
\text { 800ppm }\end{array}$ & Less safe \\
\hline More than 800ppm & Dangerous \\
\hline
\end{tabular}

Table 5: Result for CO Gas

\begin{tabular}{|l|l|}
\hline Value (ppm) & Classification result \\
\hline Less than 5ppm & Safe \\
\hline In between 5ppm \& 50ppm & Less safe \\
\hline More than 50ppm & Dangerous \\
\hline
\end{tabular}

Based on the result, e-nose is able to determine the level of dangerous of chosen gases which are LP gas and CO gas..

\section{V.CONCLUSION} several gases from the environment by using e-nose and to classify gas using classification method. Fuzzy logic application enables the system to get the optimum result. Based on the result, it is proven that e-nose system is a reliable device for human to use as the deciding factor for classifying hazardous gas in the environment. In the software part, integrating all four sensors at the same time consumed a longer time and can be considered one of the challenges. In order for them to work accurately, they need to be pre-heated for at least 30 minutes. Sometimes these sensors loss their sensitivities due to other factors such as wind. Other than that, adjusting the precise current to the LCD monitor is challenging. The LCD monitor has to be provided with
This research achieves the objectives, which are to read

explicit amount of voltage in order for it to work properly. For future improvement, other different gases can be detected by using this e-nose system such as ammonia gas, or even formaldehyde gas. Other than that, this e-nose system can be improved by having alerting functions such as by GSM module, or even a mobile application. To have a full and an almost perfect system, this e-nose system can be combined with a web-based system whereby all the data collected by the sensors can be stored in a database and they can be used for future reference for any research and development purpose.

\section{ACKNOWLEDGMENT}

This research is supported by Universiti Tenaga Nasional, Malaysia. We gratefully appreciate this support

\section{REFERENCES}

1. Hazardous Gas Definition. (2018). Retrieved from https://gasdetectionsystems.wordpress.com/2012/05/17/h azardous-gas- definition/. [Accessed: 30-Jul-2018].

2. May Levels of Toxic Gases in Gulf Back Up Claim Made by Lindsey Williams!. (2018). Retrieved from https://socioecohistory.wordpress.com/2010/06/14/may-1 evels-of-toxic-gases-in-gulf-back-up-claim-made-by-lind sey-williams-2/. [Accessed: 30-Jul-2018].

3. Zhang, D., Jiang, C., Liu, J. and Cao, Y. (2017). Carbon monoxide gas sensing at room temperature using copper oxide-decorated graphene hybrid nanocomposite prepared by layer-by-layer self-assembly. Sensors and Actuators B: Chemical, 247, pp.875-882.

4. Wang, S., Zhao, Y., Huang, J., Wang, Y., Wu, S., Zhang, S., \& Huang, W. (2006). Low- temperature carbon monoxide gas sensors based gold/tin dioxide. Solid-State Electronics, 50(11-12), 1728-1731.

5. Yu, M., Wu, R., \& Chavali, M. (2011). Effect of 'Pt' loading in $\mathrm{ZnO}-\mathrm{CuO}$ hetero-junction material sensing carbon monoxide at room temperature. Sensors And Actuators B: Chemical, 153(2), 321-328.

6. http://www.co-gassafety.co.uk/deaths.html. [Accessed: 30-Jul-2018].

7. OSHA.com - $10 \& 30$ Hour Online OSHA Outreach Training, HAZWOPER Courses. (2018). Retrieved from http://www.osha.com. [Accessed: 30-Jul-2018].

8. Hjiri, M., El Mir, L., Leonardi, S., Pistone, A., Mavilia, L., \& Neri, G. (2014). Al-doped ZnO for highly sensitive CO gas sensors. Sensors And Actuators B: Chemical, 196, 413-420.

9. Indoor Air Quality (IAQ) | US EPA. (2018). Retrieved from https://www.epa.gov/indoor-air-quality-iaq. [Accessed: 30-Jul-2018].

10. Li, N. (1993). High-Temperature Carbon Monoxide Potentiometric Sensor. Journal Of The Electrochemical Society, 140(4), 1068.

11. Paltsev, S., Zhang, D. (2015). Natural gas pricing reform in China: Getting closer to a market system?. Energy Policy, 86, 43-56.

12. National Bureau of Statistics of China. (2018). Retrieved from http://www.stats.gov.cn/english/. [Accessed: 30-Jul-2018].

13. Tarim, M. (2014). Evaluation of Burn Injuries Related to Liquefied Petroleum Gas. Journal Of Burn Care \& Research, 35(3), 159-163. 
14. D'Sa, A., \& Murthy, K. (2004). LPG as a cooking fuel option for India. Energy For Sustainable Development, 8(3), 91-106.

15. Ahuja, R., Dash, J., \& Shrivastava, P. (2011). A comparative analysis of liquefied petroleum gas (LPG) and kerosene related burns. Burns, 37(8), 1403-1410.

16. Paliwal, G., Agrawal, K., Srivastava, R., \& Sharma, S. (2014). Domestic liquefied petroleum gas: Are we using a kitchen bomb?. Burns, 40(6), 1219-1224.

17. Deshmukh, S., Bandyopadhyay, R., Bhattacharyya, N., Pandey, R., \& Jana, A. (2015). Application of electronic nose for industrial odors and gases emissions measurement and monitoring - An overview. Talanta, 144, 329-340

18. Persaud, K., \& Dodd, G. (1982). Analysis of discrimination mechanisms in the mammalian olfactory system using a model nose. Nature, 299(5881), 352-355.

19. A. Ikegami, M. Kaneyasu. (1985). Olfactory detection using integrated sensors. Proc. 3rd Int. Conf. Solid-State Sensors and Actuators (Transducers'85), Philadelphia, PA, USA. pp. 136-139.

20. What are safe levels of $\mathrm{CO}$ and $\mathrm{CO} 2$ in rooms? Kane International Limited. (n.d.). Retrieved from https://www.kane.co.uk/knowledge-centre/what-are-safelevels-of-co-and-co2-in-rooms. [Accessed: 30-Jul-2018].

21. Mamat, M., Samad, S. A., \& Hannan, M. (2011). An Electronic Nose for Reliable Measurement and Correct Classification of Beverages. Sensors, 11(6), 6435-6453.

22. Applications of Fuzzy Logic. (2007). Introduction to Fuzzy Logic Using MATLAB, 157-368.

23. Tanaka, K. (1996) An Introduction to Fuzzy Logic for Practical Applications, Springer.

24. Arshak, K., Moore, E., Lyons, G., Harris, J., \& Clifford, S. (2004). A review of gas sensors employed in electronic nose applications. Sensor Review, 24(2), 181-198.

25. The National Institute for Occupational Safety and Health (NIOSH). (2018, August 06). Retrieved from https://www.cdc.gov/niosh/index.htm. [Accessed: 30-Jul-2018].. 